This item was submitted to Loughborough's Research Repository by the author.

Items in Figshare are protected by copyright, with all rights reserved, unless otherwise indicated.

\title{
A Note on Busetti-Harvey tests for stationarity in series with structural breaks
}

PLEASE CITE THE PUBLISHED VERSION

LICENCE

CC BY-NC-ND 4.0

\section{REPOSITORY RECORD}

Harvey, David I., and Terence C. Mills. 2019. "A Note on Busetti-harvey Tests for Stationarity in Series with Structural Breaks". figshare. https://hdl.handle.net/2134/421. 
This item was submitted to Loughborough's Institutional Repository by the author and is made available under the following Creative Commons Licence conditions.

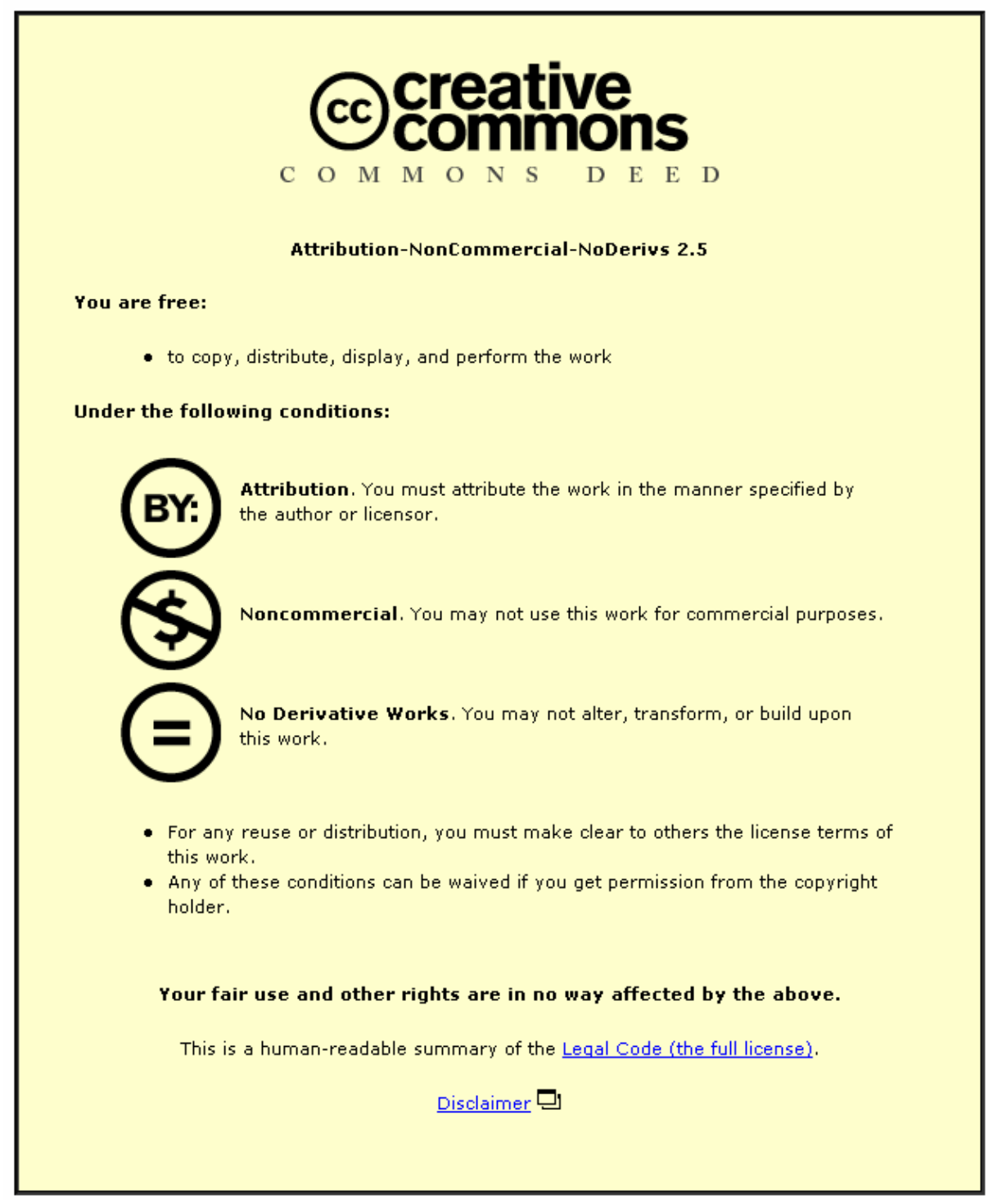

For the full text of this licence, please go to: http://creativecommons.org/licenses/by-nc-nd/2.5/ 


\title{
A NOTE ON BUSETTI-HARVEY TESTS FOR STATIONARITY IN SERIES WITH STRUCTURAL BREAKS
}

\author{
By David I. Harvey ${ }^{\dagger}$ and Terence C. Mills \\ Loughborough University
}

\begin{abstract}
In this note we highlight a minor error in the asymptotic distribution of one of the Busetti and Harvey (2001) tests for stationarity in the presence of structural breaks, and provide corrected asymptotic critical values where relevant. In addition, we examine the extent to which finite sample critical values for the Busetti-Harvey tests are approximated by their asymptotic counterparts when the location of the break is determined endogenously.
\end{abstract}

Keywords. Unit roots; endogenous breaks.

\footnotetext{
${ }^{\dagger}$ Corresponding author: Department of Economics, Loughborough University, Loughborough, Leicestershire, LE11 3TU, UK. Tel.: +44 (0)1509 222712. Email: d.i.harvey@lboro.ac.uk
} 


\section{INTRODUCTION}

Busetti and Harvey (2001) - henceforth BH-present tests of the null hypothesis of stationarity against a unit root alternative when the process contains a structural break in level and/or slope. Their methodology generalises tests by Nyblom and Mäkeläinen (1983) and Kwiatkowski et al. (1992), who present stationarity tests in the absence of such breaks. In this note, we highlight a minor error in the asymptotic distribution of one of the test statistics and present corrected asymptotic critical values where relevant. Further, for all the BH tests where the location of the break is determined endogenously, we examine how closely the asymptotic critical values approximate those in finite samples.

\section{BUSETTI-HARVEY TESTS AND CORRECTION TO ASYMPTOTIC DISTRIBUTION}

$\mathrm{BH}$ consider four models which permit different orders of deterministic components and structural breaks:

$$
\begin{array}{ll}
\text { Model } 1 & y_{t}=\mu_{t}+\delta w_{t}+\varepsilon_{t} \\
\text { Model 2 } & y_{t}=\mu_{t}+\beta t+\delta_{\mu} w_{t}+\delta_{\beta}\left(w_{t} t\right)+\varepsilon_{t} \\
\text { Model 2a } & y_{t}=\mu_{t}+\beta t+\delta_{\mu} w_{t}+\varepsilon_{t} \\
\text { Model 2b } & y_{t}=\mu_{t}+\beta t+\delta_{\beta} z_{t}+\varepsilon_{t}
\end{array}
$$

where $\mu_{t}=\mu_{t-1}+\eta_{t}$ with $\eta_{t} \sim \operatorname{NID}\left(0, \sigma_{\eta}^{2}\right), \varepsilon_{t} \sim \operatorname{IID}\left(0, \sigma^{2}\right), w_{t}=1(t>\tau)$ and $z_{t}=$ $1(t>\tau)(t-\tau)$, with $1($.) being the indicator function and $\tau$ the break point.

Model 1 has no trend component and a break in level, Model 2 contains a time trend and is subject to a simultaneous break in level and slope, while Models $2 a$ and $2 b$ also contain trends, but have breaks in level only, and slope only, respectively.

Assuming that the timing of the break is known, the locally best invariant test of $H_{0}: \sigma_{\eta}^{2}=0$ against a one-sided alternative is then

$$
\xi_{i}(\lambda)=\frac{\sum_{t=1}^{T}\left(\sum_{s=1}^{t} e_{s}\right)^{2}}{T^{2} \hat{\sigma}^{2}} \quad(i=1,2,2 a, 2 b)
$$

where $e_{t}$ denotes the residuals from the appropriate regression among (2.1)-(2.4) above, $\hat{\sigma}^{2}=T^{-1} \sum_{t=1}^{T} e_{t}^{2}$, and $\lambda=\tau / T$.

For each of the four models considered, BH present the asymptotic distribution for the test statistic $\xi_{i}(\lambda)$ under the null hypothesis. However, for Model $2 a$ (where a trend 
is included in the model but the break is in level only), there is a minor error in the distribution given. The corrected distribution is instead

$$
\xi_{2 a}(\lambda) \Rightarrow \int_{0}^{1}\left\{B_{2 a}(r, \lambda)\right\}^{2} d r
$$

where

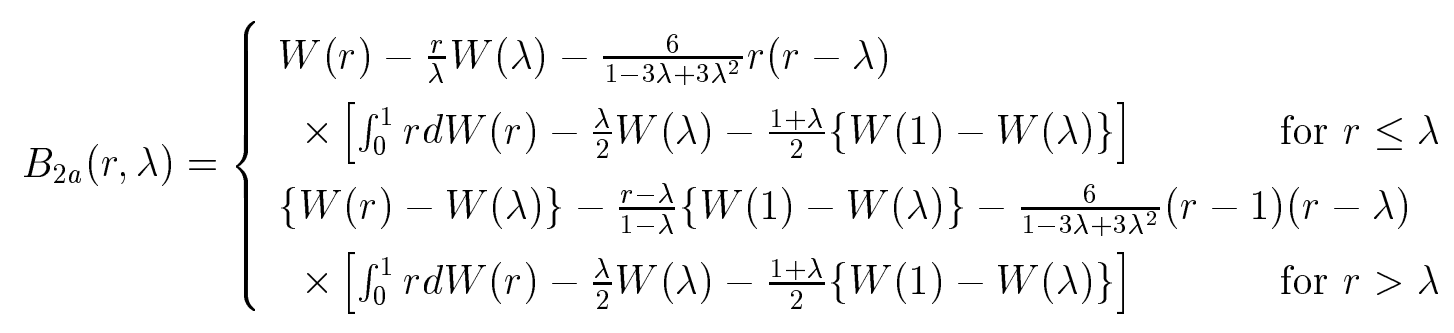

where $W($.$) is a standard Wiener process. Although the lemmas and proof provided by \mathrm{BH}$ are correct, the multiplicative factor $(r-1)$ in $B_{2 a}(r, \lambda)$ when $r>\lambda$ in $(2.7)$ is incorrectly reported as $r$ when the final expression is given. Despite this error, the asymptotic critical values when $\lambda$ is known, reported in Table I of their paper, are generated using the correct asymptotic distribution.

In contrast, however, when the location of the break is unknown, the incorrect distribution is used to generate the asymptotic critical values. When $\lambda$ is to be determined endogenously, BH recommend an approach following Zivot and Andrews (1992) where the break point is selected to give the most favourable result for the null hypothesis using the $\xi_{i}(\lambda)$ statistic of $(2.5)$, i.e.

$$
\tilde{\xi}_{i}=\inf _{\lambda \in \Lambda} \xi_{i}(\lambda) \quad(i=1,2,2 a, 2 b)
$$

where $\Lambda$ is a closed subset of the interval $(0,1)$.

Under an assumption that the magnitude of the level breaks $\left(\delta, \delta_{\mu}\right)$ decreases with the sample size at a rate faster than $T^{-1 / 2}$, and the magnitude of the slope break $\left(\delta_{\beta}\right)$ decreases faster than $T^{-3 / 2}$, the asymptotic distribution of the test statistic $\tilde{\xi}_{i}$ under the null is given by

$$
\tilde{\xi}_{i} \Rightarrow \inf _{\lambda \in \Lambda} \int_{0}^{1}\left\{B_{i}(r, \lambda)\right\}^{2} d r \quad(i=1,2,2 a, 2 b)
$$

where $B_{i}(r, \lambda)(i=1,2,2 b)$ are as defined in $\mathrm{BH}$, and $B_{2 a}(r, \lambda)$ is as defined in (2.7).

BH generate asymptotic critical values for these test statistics (reported in Table VI of their paper), but for Model $2 a$, the results make use of their incorrectly reported term $B_{2 a}(r, \lambda)$, leading to exaggerated critical values. We generated the correct critical values 
by Monte Carlo simulation of (2.9), using a sample size of $T=500$ as in BH. The space of values for $\lambda$ is restricted to a closed subset of $(0,1)$; in our simulations here and in Section 3 , we restricted $\lambda$ to lie between the conventionally chosen points $(0.2,0.8)$ and used 10000 replications. The results are given in Table I. The degree of exaggeration is quite considerable; for example, the correct $5 \%$ and $1 \%$ critical values are 0.051 and 0.068 respectively, whereas those reported by $\mathrm{BH}$ are 0.089 and 0.125 . The table also contains results for Models 1, 2 and $2 b$ using this methodology, which are of course close to those reported by $\mathrm{BH}$.

\section{FINITE SAMPLE CRITICAL VALUES}

In addition to analysing the asymptotic distribution of the test statistics, it is interesting to analyse finite sample critical values, and examine how well approximated they are by their asymptotic counterparts. Finite sample critical values for the four BH models when the break point is unknown are also provided in Table I. These critical values were simulated by repeated application of the test statistic $\tilde{\xi}_{i}$ of (2.8) to generated stationary series without breaks of length $T$ (for $T=50,100,200$ ). In each case, the critical values at different finite sample sizes are almost identical to those derived using the asymptotic distribution, even those for $T=50$, indicating that the asymptotics obtain very quickly and provide an excellent approximation of behaviour in finite samples.

\section{SUMMARY}

In summary, we have highlighted an error in the asymptotic distribution of the BH stationarity test when the model contains a trend but admits a break in level only, and presented corrected asymptotic critical values for this test when the timing of the break is determined endogenously. We have also simulated finite sample critical values of all the BH tests (again when the break point is unknown), and found that the asymptotic critical values closely approximate those in finite samples. 


\section{REFERENCES}

Busetti, F. and Harvey, A. C. (2001) Testing for the presence of a random walk in series with structural breaks. Journal of Time Series Analysis 22, 127-50.

Kwiatkowski, D., Phillips, P. C. B., Schmidt, P. and Shin, Y. (1992) Testing the null hypothesis of stationarity against the alternative of a unit root: how sure are we that economic time series have a unit root? Journal of Econometrics 44, 159-78.

Nyblom, J. and MÄKELÄINEn, T. (1983) Comparison of tests for the presence of random walk coefficients in a simple linear model. Journal of the American Statistical Association 78, 856-64.

Zivot, E. and Andrews, D. W. K. (1992) Further evidence on the Great Crash, the oilprice shock, and the unit-root hypothesis. Journal of Business and Economic Statistics 10, 251-70. 
TABLE I

Asymptotic and Finite Sample Critical Values for BH Stationarity Tests When the Break Point is Determined Endogenously

\begin{tabular}{lcccccccc}
\hline \hline & \multicolumn{3}{c}{ Model 1 } & & \multicolumn{3}{c}{ Model 2} \\
\cline { 2 - 4 } \cline { 7 - 8 } & $10 \%$ & $5 \%$ & $1 \%$ & & $10 \%$ & $5 \%$ & $1 \%$ \\
\hline$T=50$ & 0.088 & 0.107 & 0.160 & & 0.033 & 0.038 & 0.049 \\
$T=100$ & 0.086 & 0.107 & 0.158 & & 0.033 & 0.038 & 0.049 \\
$T=200$ & 0.086 & 0.108 & 0.159 & & 0.033 & 0.038 & 0.050 \\
$T=\infty$ & 0.087 & 0.108 & 0.166 & & 0.033 & 0.038 & 0.048 \\
\hline \multicolumn{4}{c}{ Model $2 a$} & & \multicolumn{3}{c}{ Model $2 b$} \\
\cline { 2 - 4 } \cline { 7 - 8 } & $10 \%$ & $5 \%$ & $1 \%$ & & $10 \%$ & $5 \%$ & $1 \%$ \\
\hline$T=50$ & 0.045 & 0.051 & 0.067 & & 0.055 & 0.064 & 0.089 \\
$T=100$ & 0.044 & 0.052 & 0.067 & & 0.056 & 0.066 & 0.090 \\
$T=200$ & 0.044 & 0.052 & 0.069 & & 0.055 & 0.067 & 0.091 \\
$T=\infty$ & 0.044 & 0.051 & 0.068 & & 0.055 & 0.065 & 0.089 \\
\hline
\end{tabular}

